\title{
Slicing Softly with Shear
}

\section{Citation}

Reyssat, E., T. Tallinen, M. Le Merrer, and L. Mahadevan. 2012. "Slicing Softly with Shear." Physical Review Letters 109 (24). https://doi.org/10.1103/physrevlett.109.244301.

\section{Permanent link}

http://nrs.harvard.edu/urn-3:HUL.InstRepos:41417328

\section{Terms of Use}

This article was downloaded from Harvard University's DASH repository, and is made available under the terms and conditions applicable to Other Posted Material, as set forth at http:// nrs.harvard.edu/urn-3:HUL.InstRepos:dash.current.terms-of-use\#LAA

\section{Share Your Story}

The Harvard community has made this article openly available.

Please share how this access benefits you. Submit a story.

Accessibility 


\title{
Slicing Softly with Shear
}

\author{
E. Reyssat, ${ }^{1, \dagger}$ T. Tallinen, ${ }^{1, \pitchfork}$ M. Le Merrer, ${ }^{1, \S}$ and L. Mahadevan ${ }^{1,2, *}$ \\ ${ }^{1}$ School of Engineering and Applied Sciences, Harvard University, Cambridge, Massachusetts 02138, USA \\ ${ }^{2}$ Department of Physics, Harvard University, Cambridge, Massachusetts 02138, USA
}

(Received 1 May 2012; published 10 December 2012)

A soft solid is more easily sliced using a combination of normal and shearing deformations rather than diced by squeezing down on it normally with the same knife. To explain why this is so, we experimentally probe the slicing and dicing of a soft agar gel with a wire, and complement this with theory and numerical simulations of cutting of a highly deformable solid. We find that purely normal deformations lead to global deformations of the soft solid, so that the blade has to penetrate deeply into the sample, well beyond the linear regime, to reach the relatively large critical stress to nucleate fracture. In contrast, a slicing motion leads to fracture nucleation with minimal deformation of the bulk and thus a much lower barrier. This transition between global and local deformations in soft solids as a function of the angle of shear explains the mechanics of the paper cut and design of guillotine blades.

Cutting is a ubiquitous process with wide ranging implications, culturally and technologically [1]. Experimenting on the kitchen table, we quickly learn that the easiest way to cut soft solids with a knife is by a slicing action, i.e., dragging the sharp blade over the soft surface without pushing too strongly into it; indeed, pushing the edge of a knife too strongly into a soft solid only squashes it. And in the office, we have all surely been the victims of the paper cut - that painful reminder of a fleeting sliding encounter between flesh and fresh paper. But beyond these everyday examples, the cutting of soft materials is of some interest in industrial food processing [2-4], and in tissue analysis in the context of histology [5], nanoskiving [6], etc. Most previous work has considered cutting as a fracture process driven by normal indentation [1-5]. Here, we focus on the transition from normal dicing to tangential slicing and highlight the role of tangential motions and forces in this process.

To quantify the cutting of a soft solid by sliding a sharp edge on its surface, we use a thin fishing line or metal wire (radius $R \approx 150 \mu \mathrm{m}$ ) held taut by clamps, like a cheese wire, to cut a piece of freshly prepared soft agar, as shown in Fig. 1(a). The shear modulus of the agar is $\mu \approx 35 \mathrm{kPa}$, and the sample is a slab of thickness $h \approx 5 \mathrm{~mm}$, length $L \approx 40 \mathrm{~mm}$, breadth $B(L \sim B>h \gg R)$. The wire is kept parallel to the cutting surface to avoid stress localization at the edge of the sample. The cutting speed (typically $0.2 \mathrm{~mm} / \mathrm{s}$ ) may be controlled by mounting the agar sample on a clamp that is moved using a servomotor. We vary the ratio $\tan \theta$ of tangential to normal speed at the surface to explore the role of shear in crack nucleation. The tangential and normal forces associated with the wire as it deforms and cuts through the surface are measured by two orthogonally mounted force sensors as shown in Fig. 1(a). The roughness of the wire may be controlled by gluing sand or glass beads on its surface or sanding it and gives us the ability to control the ratio of normal and shear stresses at the wire-agar interface.

In Figs. 1(c) and 1(d) we show the normal and tangential forces as a function of the controlled normal displacement $d$ measured relative to the undeformed agar surface. For a minimally sanded wire moving at a constant velocity normal to the agar surface, i.e., when $\theta=0$, we see that the normal force $F_{n}$ increases linearly with the deformation of the agar, i.e., $F_{n} \sim \mu d h$, until a peak force is reached before the wire breaks through the agar surface. The force then decreases to a constant steady state value that does not vary further as the wire cuts through the agar at a constant velocity. This is because once the surface has ruptured, the natural stress focusing at the advancing crack suffices to propagate the cut and occurs at a much lower value of the nominal stress. Monitoring the tangential force $F_{t}$ on the surface simultaneously shows that, while it is more than an order of magnitude smaller than $F_{n}$, it also increases with $d$ until the wire breaks through the surface and then falls nominally to a value below the noise threshold. However, as the component of slicing increases with $\theta$, the amount of shearing increases and the peak normal force falls by a factor of 5 or more [Fig. 1(e)] while the peak shearing force increases, still remaining below the normal force [Fig. 1(f)]. We thus see clearly that cutting involves two processes: a nucleation stage dominated by the nonlocal large scale deformation of the gel without fracture or failure until a critical stress for fracture nucleation is reached. The nominal stress then falls to a much smaller value and remains constant during the growth stage when the cut progresses steadily as the wire deforms the gel only locally. We see that as $\theta$ is increased, the shearing motions greatly reduce the fracture nucleation barrier, so that it becomes easy to slice through a soft solid. In our experiments, we find that the slicing mode crucially changes the initiation of fracture but has no advantage after the fracture has initiated; others 
(a)

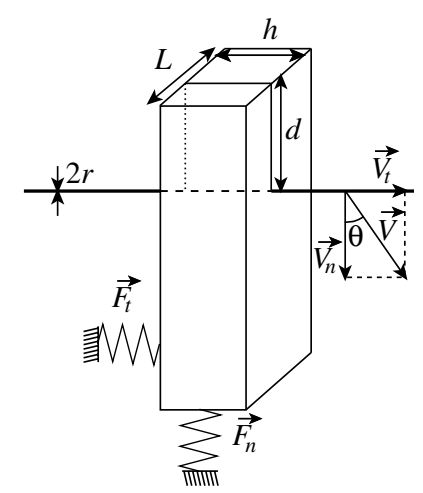

(b)
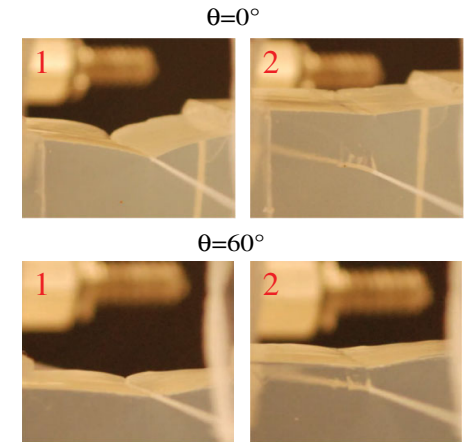

(c)

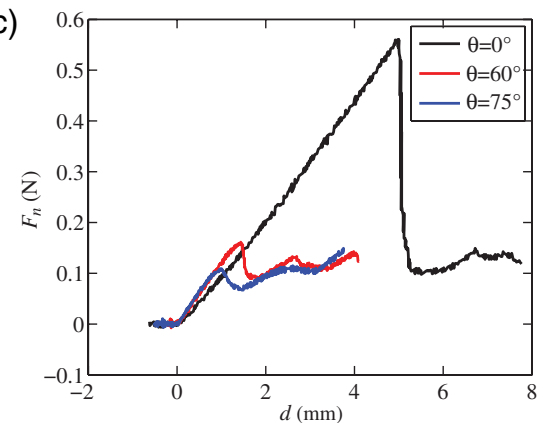

(d)

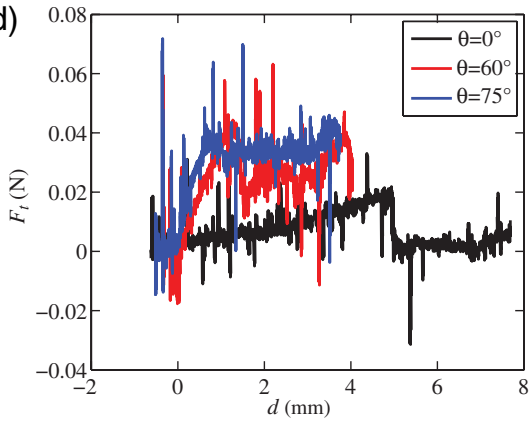

(e)

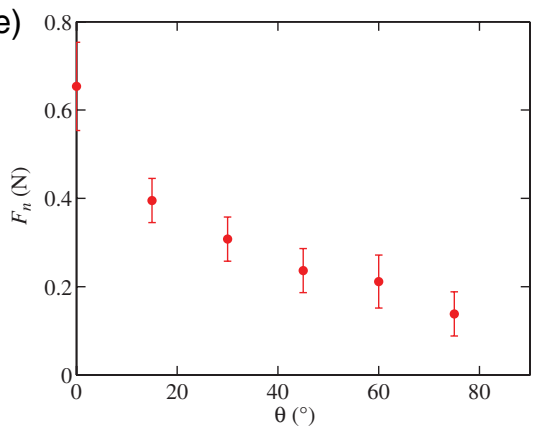

(f)

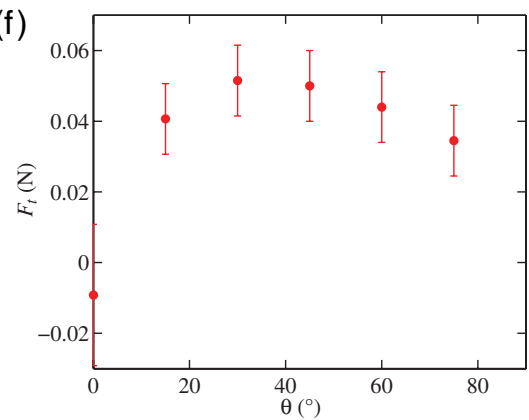

FIG. 1 (color online). (a) Schematic of cutting experiment. A wire of radius $R \approx 150 \mu \mathrm{m}$ is used to cut through a piece of soft gel $(\mu \approx 35 \mathrm{kPa}$ ) with a velocity $V$. The thickness of the sample is $h \approx 5 \mathrm{~mm}$. Shear is controlled via the kinematic angle $\theta$ between the direction of penetration by the wire and the normal to the surface of the gel: the normal velocity is kept constant $\left(V_{n}=0.2 \mathrm{~mm} / \mathrm{s}\right)$, while the tangential component $V_{t}$ varies as $\theta$ changes. (b) The gel surface deforms strongly before the initiation of penetration, and then rebounds partially; these phases correspond to the nucleation and growth phases of the fracture for purely normal indentation (top) and increased shearing motion (bottom). We see that shearing reduces the maximum deformation of the gel before fracture is nucleated. (c) The normal force $F_{n}$ as a function of penetration depth for various angles of cutting $\theta$ characterizing the relative rate of shearing. (d) The tangential shear force $F_{t}$ as a function of penetration depth for various angles of cutting $\theta$. (e) The peak normal force $F_{n}^{m}$ as a function of the shear angle $\theta$ for a fixed normal velocity drops by a factor of about 5 as the shear angle increases from $0^{\circ}$ to $75^{\circ}$. (f) The peak shear force $F_{t}^{m}$ as a function of the shear angle $\theta$ for the same normal displacement rate shows a shallow maximum but at a value that is nearly an order of magnitude smaller than the normal force.

have argued [7] that slicing reduces the force required in the postfracture stage, although the effect is fairly weak and not noticeable in our experiments.

To understand the mechanics of crack nucleation, we consider the plane problem of line loading of an elastic half-space. Assuming cylindrical coordinates with the $z$ axis at the surface of the half-space, angle $\phi=0$ normal to the surface, and the line $r=0$ loaded by a force of magnitude $f$ per unit length, with components $f_{n}$ in the normal direction and $f_{t}$ along the $z$ axis, the resulting stress tensor can be obtained analytically using the theory of contact mechanics [8] and yields

$$
\boldsymbol{\sigma}=\left[\begin{array}{ccc}
\sigma_{r r} & 0 & \sigma_{r z} \\
0 & 0 & 0 \\
\sigma_{z r} & 0 & \sigma_{z z}
\end{array}\right],
$$

where

$$
\begin{gathered}
\sigma_{r r}=2 \sigma_{z z}=-\frac{2 f_{n} \cos \phi}{\pi r}, \\
\sigma_{r z}=\sigma_{z r}=\frac{f_{t}}{\pi r} .
\end{gathered}
$$

The general combination of tangential and normal stresses implies that the highest tensile stress induced by line loading is given by the largest eigenvalue of the stress tensor and is given by Eq. (1):

$$
\sigma_{1}=-\frac{3 f_{n} \cos \phi}{2 \pi r}+\sqrt{\left(\frac{f_{n} \cos \phi}{2 \pi r}\right)^{2}+\left(\frac{f_{t}}{\pi r}\right)^{2}} .
$$

Typically soft solids can resist large compressive stresses but fail under critical tensile stresses that might result from a combination of compression and/or shear [9]. For pure normal loading $\left(f_{t}=0\right) \sigma_{1} \leq 0$ for any $\phi$, which means that the stress is compressive everywhere. 
For combined normal and tangential loading, however, we find that a tensile stress arises with the maximum $\sigma_{1}=\frac{f_{t}}{\pi r}$ at the surface of the half-space $(\phi= \pm \pi / 2)$; this is the qualitative origin of the effective efficacy of tangential slicing relative to normal dicing as we shall see.

Our simple linear line-loading approach, while useful, is only suggestive as gels and biomaterials are tough and can thus deform strongly before nucleation of fracture. Similarly, the assumption of contact mechanics that requires the radii of contacting bodies to be relatively well separated or very similar [8] is also invalid in general. To test the validity of our minimal model, we resort to numerical simulations of varying complexity to mimic the cutting of a slab, which is itself modeled as a neo-Hookean material [10], being cut by a rigid wire of radius $R$. Discretizing our slab using tetrahedral elements arranged in a cubical lattice, our discrete deformation gradient $\mathbf{F}$ is determined from the deformed basis vectors of each tetrahedron, while the stress $\boldsymbol{\sigma}=\frac{1}{J} \frac{\partial W}{\partial \mathbf{F}} \mathbf{F}^{\mathrm{T}}$ is derived from the neo-Hookean strain energy density $W=$ $\frac{\mu}{2}\left[\operatorname{Tr}\left(\mathbf{F F}^{\mathrm{T}}\right) J^{-2 / 3}-3\right]+\frac{K}{2}(J-1)^{2}$ (see Ref. [10]), where $\mu$ and $K$ are the shear and bulk modulus, respectively, and $J=\operatorname{det}(\mathbf{F})$. We assume that the bulk modulus, $K=30 \mu$, is much larger than the shear modulus, a reasonable approximation for gels and rubbers that are nearly incompressible. To determine the nodal forces, we note that the stresses in a tetrahedron produces tractions on its surfaces, which are then integrated to produce the resultants acting on its nodes. The energy of the slab is minimized by using damped Newtonian dynamics of the nodes. In all simulations the base of the slab is clamped and the sides are constrained to move only in the vertical direction, while the surface not in contact with the wire is assumed to be free.

Our first model of the slab assumes that its size $L=$ $B=50 R$ with periodicity in the direction of the wire (Fig. 2), so that it does not account for the role of the lateral edges which first contact the cutting wire. Our numerical lattice is assumed to have $400 \times 300 \times 3$ nodes (length $\times$ breadth $\times$ thickness) with periodic boundary conditions in the thickness direction. The normal contact between the wire and the slab is modeled as a linear repulsive force in the radial direction of the wire. To mimic the tangential motions associated with slicing, lattice nodes in contact are given forces in the direction of the $z$ axis, with magnitudes proportional to the radial contact forces such that a particular value of the ratio of tangential to normal forces $f_{t} / f_{n}$ is achieved; this is similar to a simple Amontons-type frictional law at the interface. For small values of the scaled nominal stress resultant $f / \mu R$, our simulated stress distributions agree well with the simple analytic theory: cutting attempted using purely normal stress resultants leads predominantly to a compression of the slab, while including a shearing or slicing component leads to tensile stresses at its surface. Of course, the finite
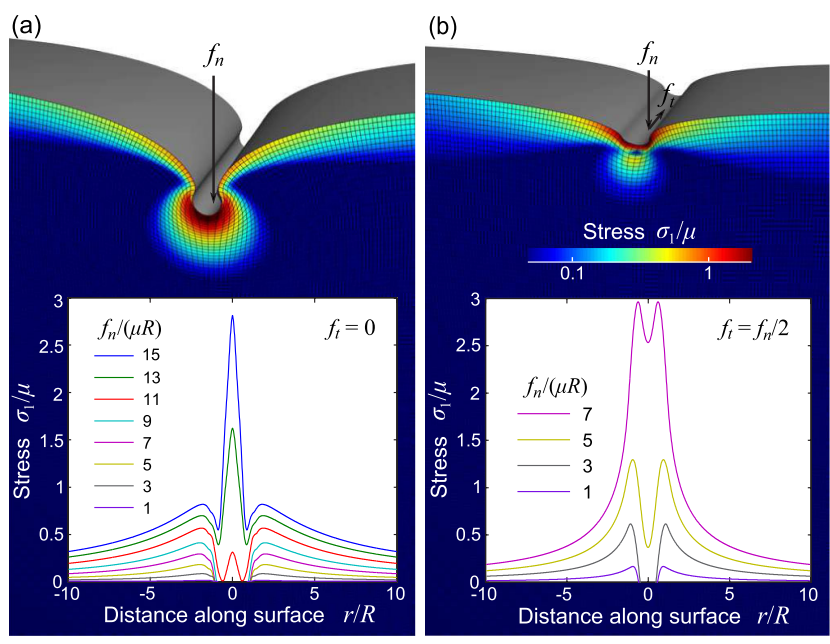

FIG. 2 (color online). Numerical simulations of periodic slabs with shear modulus $\mu$ that are squeezed or sheared by a wire of radius $R$. In (a) the slab is squeezed normally by a force $f_{n}=15 \mu R$ per unit length. In (b) $f_{n}=7 \mu R$ and the slab is, in addition, sheared tangentially by $f_{t}=f_{n} / 2$. Color indicates the magnitude of the largest principal (tensile) stress $\sigma_{1}$. The insets show tensile stress at the surface of the slab, for various $f_{n}$, as a function of distance $r$ from the middle line in the undeformed configuration.

radius of the wire smooths out the singularity of the line-loading solution so that the maximum tensile stress $\sigma_{1} \sim f_{t} / R$, which decays as $\sigma_{1} \sim 1 / r$ as is apparent for $r>R$ [Fig. 2(b)]. For larger values of $f_{n} / \mu R$, however, nonlinear effects can and do lead to tensile stresses even in purely normal loading, due to the fact that the surface stretches in a direction perpendicular to the wire and along the free surface [Fig. 2(a)]. However, this effect becomes significant only when penetration of the wire is comparable to or larger than its radius. Furthermore, the location of the region of maximum tensile stress also changes with the nominal state of surface stress. For the case of purely normal loading, the maximum tensile stress is first found along the wings of the wire-slab contact zone, while for $f_{n} / \mu R \gtrsim 12$ the maximum moves right below the wire, along the line of loading symmetry, as shown in Fig. 2(a). In contrast, in the case when there is both normal and shear loading, the stress maximum remains along the wings of the contact zone for the entire range of forces studied, as shown in Fig. 2(b). Just as in our experiments, our simulations show that purely normal loading requires the wire to indent into the slab deeply to reach a large tensile stress [Fig. 2(a)], whereas shear loading allows us to reach the same local critical stress using a much smaller displacement and force [Fig. 2(b)]. Thus, for a given critical fracture nucleation stress, cutting by slicing action requires only a fraction of the force associated with pure normal loading.

To account for the three-dimensional nature of the problem, particularly in the context of how the wire first 
(a)

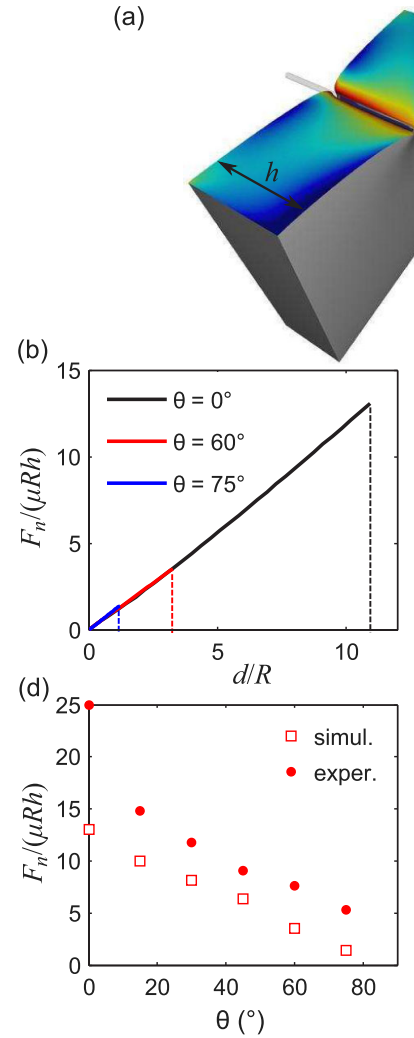

characteristic behavior with the change in the angle $\theta$ in our simulations as in our experiments [Figs. 3(d) and 3(e)]. The normal force decreases as shear increases, whereas the tangential force shows a maximum $F_{t} \approx 2 \mu R h$ comparable to its experimental counterpart. Relative normal forces were, however, slightly higher in the experiments. The cause of this mismatch may be that friction at the interface remains poorly quantified. Totally suppressing friction would lead to sliding of the blade on the surface sample, and no tangential force, thus suppressing the benefit of shearing, as is observed with smooth cutting wires. For any finite friction, the optimal configuration will then be one poised at the edge of sliding just as fracture nucleation occurs; indeed, any sliding leads to retarded fracture nucleation and increases the elastic energy stored in the solid before cutting actually starts.

Our study demonstrates the role of normal and tangential loading on changing the effective nucleation barrier for cutting by the interconversion of global and local modes of deformation on the surface of a soft solid. Since any stored energy in a strongly deformed soft solid is irreversibly lost when the stress reaches the critical fracture threshold, the thermodynamic work of cohesion provides only a poor lower bound on the energy of fracture [9]. Here we see an application of this principle wherein the fracture nucleation barrier is reduced by converting the global deformation of the solid to a local one using the simple geometry of loading. This occurs through the slicing action of a blade which creates a critical local tension at the surface with minimal global compression of the bulk, while pure normal loading involves strong global deformation before fracture initiation and leads to material damage. In practice, sliding might limit the efficiency of this process, so that when cutting soft solids, we must impose a minimal normal force that enables fracture nucleation due to slicing. The same principle that holds for paper cuts also plays a role in the macabre guillotine: the angled blade is designed to slice through flesh sharply and do so with minimum resistance rather than squeeze it.

We thank the Harvard-NSF MRSEC and the MacArthur Foundation for partial support. $400 \times 300 \times 100$ nodes (length $\times$ breadth $\times$ thickne Again, as in our experiments, we control the tangential and normal velocities of the wire and apply a no-slip condition between the wire and the slab. By comparing Figs. 3(b) and 3(c) and Figs. 1(c) and 1(d), we find that indentation force profiles obtained using simulations are very similar to those seen in our experiments. After the slight nonlinear stiffening at $d \lesssim R$, the slope of the normal force is well approximated $F_{n} \approx 5 \mu d h / \log (B / R)$, where the logarithmic denominator is a consequence of the $\sigma \sim$ $1 / r$ far field decay of the dominantly two-dimensional stress field. By assuming that fracture is nucleated for a tensile surface stress $\sigma^{*}=2 \mu$, we find estimates for cutting forces that are comparable to the experimentally measured forces at fracture nucleation. Furthermore, we find that the normal and tangential forces at nucleation show the same

*1m@seas.harvard.edu

${ }^{\dagger}$ Present address: PMMH, UMR 7636 du CNRS, ESPCI, 10 rue Vauquelin, 75005 Paris, France, EU.

${ }^{*}$ Present address: Department of Physics, University of Jyväskylä, P.O.Box 35, FI-40014 Jyväskylä, Finland.

§Present address: INSP, UMR 7588 du CNRS, Université Paris 6, 4 place Jussieu, 75005 Paris, France, EU.

[1] A. G. Atkins, The Science and Engineering of Cutting (Elsevier, New York, 2009).

[2] D. Zhou and G. McMurray, Robotica 28, 311 (2010).

[3] I. Camyab, S. Chalranarti, and J. Williams, J. Mater. Sci. 33, 2763 (1998). 
[4] S. M. Goh, M. N. Charalambides, and J. G. Williams, Eng. Fract. Mech. 72, 931 (2005).

[5] M. Mahvash and V. Hayward, Haptics-e 2, 1 (2001).

[6] Q. Xu, R. M. Rioux, M. D. Dickey, and G. M. Whitesides, Acc. Chem. Res. 41, 1566 (2008).

[7] A. G. Atkins, X. Xu, and G. Jeronimidis, J. Mater. Sci. 39, 2761 (2004).
[8] K. L. Johnson, Contact Mechanics (Cambridge University Press, Cambridge, England, 1987).

[9] G. J. Lake and A. G. Thomas, Proc. R. Soc. B 300, 108 (1967).

[10] L. R. G. Treloar, The Physics of Rubber Elasticity (Oxford University Press, New York, 1975). 